\title{
ORDER SUMMABILITY OF MULTIPLE FOURIER SERIES
}

\author{
BY \\ G. E. PETERSON AND G. V. WELLAND( $\left.{ }^{1}\right)$
}

\begin{abstract}
Jurkat and Peyerimhoff have characterized monotone Fouriereffective summability methods as those which are stronger than logarithmic order summability. Here the analogous result for double Fourier series is obtained assuming unrestricted rectangular convergence. It is also shown that there is a class of order summability methods, which are weaker than any Cesàro method, for which the double Fourier series of any $f \in L$ is restrictedly summable almost everywhere. Finally, it is shown that square logarithmic order summability has the localization property for exponentially integrable functions.
\end{abstract}

1. Introduction. In [3] and [4] Jurkat and Peyerimhoff defined logarithmic order summability $L_{1}^{*}$, and showed that a monotone summability method $M$ is Fourier-effective if and only if $M \supseteq L_{1}^{*}$. Here we obtain the analogous result in two dimensions (Theorem 7.1), and answer other questions pertaining to order summability which are peculiar to multiple Fourier series.

In particular, we obtain the analogues in two dimensions of [3, Theorems 1.1, 1.2, 4.1] and [4, Theorems 1.1 and 1.2]. That is, we characterize $F_{C}$-effective methods by properties of their kernels (Theorem 9.1), and give useful necessary conditions for effectiveness in terms of the matrix elements (Theorem 4.2). We show that the Fourier series for any $f \in L \log ^{+} L$ is unrestrictedly rectangular summable $L_{1}^{*}$ to $f$ at any Lebesgue point for which the maximal function of $f$ is finite (Theorem 5.1). We also give certain sufficient conditions for the order summability method $[g]$ to be included in the matrix method $A$ (Theorem 7.2).

In addition to obtaining these analogous results, we prove that the double Fourier series for $f \in L$ is restrictedly summable $L_{\alpha}^{*}$ to $f$ almost everywhere for $\alpha>1$ (Theorem 6.1). A similar result for Cesàro methods was obtained in [5]. This result is reproduced in [8, p. 311], for the $C_{1}$ method. Since $L_{\alpha}^{*}$ is strictly included in $\bigcap_{\beta>0} C_{\beta}$ for every $\alpha>1$ [4, p. 257], our result improves the result of [5].

Received by the editors January 24, 1974.

AMS (MOS) subject classifications (1970). Primary 42A24, 40B05; Secondary 42A62, 40D25.

Key words and phrases. Logarithmic order summability, multiple Fourier series, order summability, restricted rectangular convergence, localization, exponentially integrable, maximal function.

(1) Supported in part by NSF grant GP-34630. 
Finally, we show that square $L_{1}^{*}$ summability has the localization property for exponentially integrable functions. This plays the role of an "end point" result for a theorem proved by Igari [2] in which it is proved that square $C_{\alpha}$ summability has the localization property for $f \in L^{p}$ if $p \geqslant(n-1) / \alpha$ but not if $p<(n-1) / \alpha$. Our result corresponds to the limiting case as $p$ tends to $\infty$ and $\alpha$ tends to 0 .

For convenience, we will work in two dimensions for all cases except for that of the localization result. In most cases it will be clear that similar results are valid if the dimension, $d$, is greater than two.

2. Basics. We will use $m, n, \cdots$ etc. to denote either integers or $d$-tuples with integer coordinates. The context will make clear which is meant. Notation for real numbers and real vectors will be handled similarly with letters $x, y, \cdots$ etc.

Let $\Lambda_{d}$ be the lattice of $d$-tuples with integer coordinates and $\Lambda_{d}^{+}=$ $\left\{m \in \Lambda_{d} \mid m_{i} \geqslant 0, i=1,2, \cdots, d\right\}$. Let

$$
\hat{f}(m)=(1 / 2 \pi)^{d} \int_{T^{d}} f(x) e^{-i m \cdot x} d x, \quad m \in \Lambda_{d},
$$

where $T^{d}=[-\pi, \pi]^{d}$ and $f \in L\left(T^{d}\right)$. Let $k \in \Lambda_{d}^{+}$and set $S_{k}(x, f)=$ $\Sigma \hat{f}(m) e^{i m \cdot x}$ where the sum is over all $m \in \Lambda_{d}$ for which $\left|m_{i}\right| \leqslant k_{i}, i=$ $1,2, \cdots, d$. Suppose $g(x) \geqslant 1$ for all $x \in[0, \infty)^{d}$ and define for any "sequence" $\left\{s_{k}\right\}, k \in \Lambda_{d}^{+}$,

$$
\sigma_{n, m}=\frac{1}{g(m /(n+1))} \frac{1}{*(n+1-m)} \sum\left(s_{k}-s\right) ; \quad n, m \in \Lambda_{d}^{+},
$$

where

$$
\frac{m}{n+1}=\left(\frac{m_{1}}{n_{1}+1}, \cdots, \frac{m_{d}}{n_{d}+1}\right), \quad n+1=\left(n_{1}+1, \cdots, n_{d}+1\right),
$$

${ }^{*} x=x_{1} \cdot x_{2} \cdots x_{d}$, for any vector $x$, and the summation runs over all $k=\left(k_{1}, k_{2}\right.$, $\left.\cdots, k_{d}\right)$ for which $m_{j} \leqslant k_{j} \leqslant n_{j}, j=1,2, \cdots, d$. When $s_{k}=S_{k}(x, f)$ and $s=f(x)$, then $\sigma_{n, m}$ becomes $\sigma_{n, m}(x, f)$.

We will say that $\left\{s_{k}\right\}$ is unrestrictedly rectangular order summable $[g]$ to $s$ if $\sigma_{n, m}$ tends to zero as $n$ tends to infinity uniformly in $m, 0 \leqslant m \leqslant n$. That is, for any $\epsilon>0$ there exists $M>0$ such that $\left|\sigma_{n, m}\right|<\epsilon$ whenever $0 \leqslant m \leqslant n$ and $n_{i} \geqslant M$ for $i=1,2, \cdots, d$. (For any pair of vectors, $x$ and $y$, by $x \leqslant y$ we mean $x_{i} \leqslant y_{i}, i=1,2, \cdots, d$.) The summability will be called restricted if a constant $c \geqslant 1$ is specified and $n$ tends to infinity under the restrictions 


$$
\frac{1}{c} \leqslant \frac{n_{j}+1+m_{j}}{n_{i}+1+m_{i}} \leqslant c \text { and } \frac{1}{c} \leqslant \frac{n_{j}+1-m_{j}}{n_{i}+1-m_{i}} \leqslant c
$$

for $i, j=1,2, \cdots, d$. The summability is called square if the constant, $c$ of (2.1), is one. Again in both of these, convergence of $\sigma_{n, m}$ to zero is to be uniform in $m, 0 \leqslant m \leqslant n$.

When

$$
g(t)=g\left(t_{1}, \cdots, t_{d}\right)=\left(1+\log 1 /\left(1-t_{1}\right)\right)^{\alpha} \cdots\left(1+\log 1 /\left(1-t_{d}\right)\right)^{\alpha}
$$

we obtain the summability method $L_{\alpha}^{*}$. We denote the corresponding $\sigma_{n, m}$ by $\sigma_{n, m}^{\alpha}$.

For integers $n$ and $m(0 \leqslant m \leqslant n)$ and real $t$, define

$$
K_{n, m}(t)=\frac{1}{n+1-m} \frac{\sin (n+1+m) t / 2 \sin (n+1-m) t / 2}{\sin ^{2} t / 2} .
$$

Elementary estimates show that for $0 \leqslant t \leqslant \pi$ we have

$$
\begin{aligned}
& \left|K_{n, m}(t)\right| \leqslant(n+1+m) \pi^{2} / 4, \\
& \left|K_{n, m}(t)\right| \leqslant \pi^{2} / 2 t, \\
& \left|K_{n, m}(t)\right| \leqslant \pi^{2} /(n+1-m) t^{2} .
\end{aligned}
$$

Let $\epsilon=(n+1+m)^{-1}$ and $\delta=(n+1-m)^{-1}$. Often, in what follows (2.3) will be used for $t \in[0, \epsilon),(2.4)$ for $t \in[\epsilon, \delta)$ and (2.5) for $t \in[\delta, \pi]$.

A short calculation shows that $(n, m$ and $t$ are now vectors)

$$
\sigma_{n, m}^{1}(x, f)=\frac{1}{\pi^{d}} \cdot \frac{1}{g(m /(n+1))} \cdot \int_{Q^{d}}[f(x+t)-f(x)] \bar{K}_{n, m}(t) d t
$$

where $Q^{d}=[0, \pi]^{d}$ and $\bar{K}_{n, m}(t)=K_{n_{1}, m_{1}}\left(t_{1}\right) \cdots K_{n_{d}, m_{d}}\left(t_{d}\right)$.

The letter $C$ will stand for a constant which is independent of any of the important parameters or functions involved, and it will not necessarily be the same constant at each occurence.

3. Fourier effectiveness. Let $f \in L[-\pi, \pi]^{2}$ have the Fourier expansion $f(x) \sim \Sigma \hat{f}(m) e^{i m \cdot x}$. Let

$$
\phi_{x}(t)=1 / 4\left[f(x+t)+f\left(x_{1}+t_{1}, x_{2}-t_{2}\right)+f\left(x_{1}-t_{1}, x_{2}+t_{2}\right)+f(x-t)\right] .
$$

Then $\phi_{x} \in L[0, \pi]^{2}$ and has the Fourier-cosine expansion

$$
\phi_{x}(t) \sim \sum_{n>0} a_{n}(x) \cos n_{1} t_{1} \cos n_{2} t_{2}
$$


where

$$
\begin{aligned}
a_{n}(x)= & \hat{f}(n) e^{i n \cdot x}+\hat{f}\left(-n_{1}, n_{2}\right) e^{i\left(-n_{1} x_{1}+n_{2} x_{2}\right)} \\
& +\hat{f}\left(n_{1},-n_{2}\right) e^{i\left(n_{1} x_{1}-n_{2} x_{2}\right)}+\hat{f}(-n) e^{-i n \cdot x}, \quad n_{1} \neq 0, n_{2} \neq 0, \\
a_{\left(n_{1}, 0\right)}(x)= & \hat{f}\left(n_{1}, 0\right) e^{i n_{1} x_{1}}+\hat{f}\left(-n_{1}, 0\right) e^{-i n_{1} x_{1}}, \quad n_{1} \neq 0, \\
a_{\left(0, n_{2}\right)}(x)= & \hat{f}\left(0, n_{2}\right) e^{i n_{2} x_{2}}+\hat{f}\left(0,-n_{2}\right) e^{-i n_{2} x_{2}}, \quad n_{2} \neq 0, \\
a_{(0,0)}(x)= & \hat{f}(0) .
\end{aligned}
$$

By $F_{C}$ we denote the class of all series $\Sigma a_{n}(x)$ for which $\phi_{x}(t)$ is continuous at $t=0$. Similarly, $F_{L}$ denotes the class of all series $\Sigma a_{n}(x)$ such that the maximal function of $\phi_{x}, M \phi_{x}$, is finite at $t=0$ and $t=0$ is a Lebesgue point of $\phi_{x}$. The maximal function of a function $\phi$ is defined by

$$
(M \phi)(t)=\sup \left\{\frac{1}{h_{1} h_{2}} \int_{0}^{h_{1}} \int_{0}^{h_{2}}|\phi(u+t)| d u: h_{1}>0, h_{2}>0\right\}
$$

and a Lebesgue point of $\phi$ is a point $t$ for which

$$
\lim _{h_{1} \rightarrow 0, h_{2} \rightarrow 0} \frac{1}{h_{1} h_{2}} \int_{0}^{h_{1}} \int_{0}^{h_{2}}|\phi(u+t)-\phi(t)| d u=0 .
$$

In one dimension, if $t=0$ is a Lebesgue point for a periodic $\phi \in L$, then $(M \phi)(0)<\infty$. However in higher dimensions this need not be true. In [6], it is shown that for a function

$$
\phi \in L\left(\log ^{+} L\right)^{d-1}=\left\{\phi: \int|\phi|\left(1+\log ^{+}|\phi|\right)^{d-1}<\infty\right\}
$$

$\left(\log ^{+}|x|=0\right.$ for $|x| \leqslant 1$, and $\log ^{+}|x|=\log |x|$ for $\left.|x|>1\right)$ that both $(M \phi)(t)<\infty$ and $t$ is a Lebesgue point hold for almost every $t$.

In what follows, the point $x$ will be arbitrary but fixed unless otherwise indicated and it will usually be dropped from the notation.

Consider a two-dimensional summability method $B=\left(b_{n v}\right)$ in the seriesto-sequence form which satisfies $\left(n, \nu \in \Lambda_{2}^{+}\right.$and $n \rightarrow \infty$ means unrestricted)

$$
\begin{aligned}
& b_{n \nu} \rightarrow 1 \quad(n \rightarrow \infty, \nu \text { fixed }), \\
& b_{n \nu} \rightarrow 0 \quad\left(n \text { fixed, } \nu_{1} \rightarrow \infty \text { or } \nu_{2} \rightarrow \infty\right) .
\end{aligned}
$$

Assume also that

$$
\sigma_{n}(\phi)=\sum_{\nu>0} b_{n \nu} a_{\nu} \quad\left(C_{1}\right)
$$


that is, that $\sigma_{n}(\phi)$ exists for each $n$ in the Cesàro $C_{1}$-sense for all series $\Sigma a_{\nu}$ in $F_{C}$, respectively in $F_{L}$. Finally, assume that

$$
\sigma_{n}(\phi) \rightarrow s=\phi(0) \quad(n \rightarrow \infty)
$$

for all $\phi$ corresponding to series in $F_{C}$, respectively in $F_{L}$.

A method $B$ satisfying (3.1), (3.2), (3.3) and (3.4) is called Fouriereffective, or more precisely $F_{C}$-effective, respectively $F_{L}$-effective.

For a measurable set $E$, of finite measure, $|E|$ denotes its Lebesgue measure.

4. $F_{C}$ effectiveness. In this section we note the following theorem which has a proof essentially the same as that of [3, Theorem 1.1]. We specifically include it because it leads to one of the central conditions of the paper.

THEOREM 4.1. A method $B=\left(b_{n v}\right)$ is $F_{C^{-}}$effective if and only if

$$
\begin{aligned}
\frac{1}{4} b_{n 0} & +\frac{1}{2} \sum_{\nu_{1}=0}^{\infty} b_{n\left(\nu_{1}, 0\right)} \cos \nu_{1} t_{1} \\
& +\frac{1}{2} \sum_{\nu_{2}=0}^{\infty} b_{n\left(0, \nu_{2}\right)} \cos \nu_{2} t_{2}+\sum_{\nu>0} b_{n \nu} \cos \nu_{1} t_{1} \cos \nu_{2} t_{2} \quad(n \geqslant 0)
\end{aligned}
$$

are the Fourier-cosine expansions of functions $b_{n} \in L[0, \pi]^{2}$ satisfying, for every $\delta, 0 \leqslant \delta \leqslant \pi$,

$$
\underset{t \in T_{\delta}}{\text { ess sup }}\left|b_{n}(t)\right| \leqslant M_{\delta} \quad(n \geqslant 0),
$$

where $T_{\delta}=[0, \pi]^{2}-[0, \delta]^{2}$,

$$
\begin{gathered}
\int_{0}^{\pi} \int_{0}^{\pi}\left|b_{n}(t)\right| d t \leqslant M \quad(n \geqslant 0), \\
\int_{T_{\delta}} b_{n}(t) d t \rightarrow 0 \quad(n \rightarrow \infty), \\
\left(\frac{2}{\pi}\right)^{2} \int_{0}^{\pi} \int_{0}^{\pi} b_{n}(t) d t \rightarrow 1 \quad(n \rightarrow \infty) .
\end{gathered}
$$

Since $F_{C} \subseteq F_{L}$, trivially, $F_{L}$-effectiveness implies $F_{C}$-effectiveness. Therefore, conditions (4.1) to (4.4) are also necessary for $F_{L}$-effectiveness.

Theorem 4.2. If $B$ is $F_{C}$-effective or $F_{L}$-effective then

$$
\left|\sum_{\nu_{1}=0, \nu_{1} \neq k_{1}}^{2 k_{1}} \sum_{\nu_{2}=0, \nu_{2} \neq k_{2}}^{2 k_{2}} \frac{b_{n \nu}}{\left(k_{1}-\nu_{1}\right)\left(k_{2}-\nu_{2}\right)}\right| \leqslant M
$$


where $M$ is a constant independent of $n$ and $k$. If, in addition,

$$
\int_{T_{\delta}}\left|b_{n}(t)\right| d t \rightarrow 0 \quad(n \rightarrow \infty)
$$

then

(4.7a) $\lim _{n \rightarrow \infty} \sup _{k_{2}} \sum_{\nu_{1}=0, \nu_{1} \neq k_{1}}^{2 k_{1}} \sum_{\nu_{2}=0, \nu_{2} \neq k_{2}}^{2 k_{2}} \frac{b_{n \nu}}{\left(k_{1}-\nu_{1}\right)\left(k_{2}-\nu_{2}\right)}=0$ for each $k_{1}$,

(4.7b) $\lim _{n \rightarrow \infty} \sup _{k_{1}} \sum_{\nu_{1}=0, \nu_{1} \neq k_{1}}^{2 k_{1}} \sum_{\nu_{2}=0, \nu_{2} \neq k_{2}}^{2 k_{2}} \frac{b_{n v}}{\left(k_{1}-\nu_{1}\right)\left(k_{2}-\nu_{2}\right)}=0$ for each $k_{2}$.

Proof. Let

$$
p_{k}(t)=\sum_{m=1}^{k} \frac{1}{m}\{\cos (k-m) t-\cos (k+m) t\}=2 \sin k t \sum_{m=1}^{k} \frac{\sin m t}{m}
$$

and note that $p_{k}(t)$ is uniformly bounded in $k$ and $t$. For (4.5) put $f_{k}\left(t_{1}, t_{2}\right)=p_{k_{1}}\left(t_{1}\right) p_{k_{2}}\left(t_{2}\right)$. Then (4.2) implies

$$
\sum_{\nu_{1}=0, \nu_{1} \neq k_{1}}^{2 k_{1}} \sum_{\nu_{2}=0, \nu_{2} \neq k_{2}}^{2 k_{2}} \frac{b_{n \nu}}{\left(k_{1}-\nu_{1}\right)\left(k_{2}-\nu_{2}\right)}=\left(\frac{2}{\pi}\right)^{2} \int_{0}^{\pi} \int_{0}^{\pi} f_{k}(t) b_{n}(t) d t=O(1) .
$$

For (4.7) we consider (4.7a) only since (4.7b) is similar. Let

$$
M=\sup \left\{\left|p_{k_{2}}\left(t_{2}\right)\right| \frac{4}{\pi^{2}} \int_{0}^{\pi} \int_{0}^{\pi}\left|b_{n}(t)\right| d t: k_{2} \geqslant 1, t_{2} \in R\right\} .
$$

Let $\epsilon>0$ be given and choose $\delta>0$ such that $\left|p_{k_{1}}\left(t_{1}\right)\right|<\epsilon(2 M)^{-1}$ for $0 \leqslant t_{1} \leqslant \delta$. Then

$$
\begin{aligned}
& \left|\sum_{\nu_{1}=0, \nu_{1} \neq k_{1}}^{2 k_{1}} \sum_{\nu_{2}=0, \nu_{2} \neq k_{2}}^{2 k_{2}} \frac{b_{n \nu}}{\left(k_{1}-v_{1}\right)\left(k_{2}-v_{2}\right)}\right|=\left|\frac{4}{\pi^{2}} \int_{0}^{\pi} \int_{0}^{\pi} f_{k}(t) b_{n}(t) d t\right| \\
& \leqslant \frac{4}{\pi^{2}} \frac{\epsilon}{2 M} \int_{0}^{\delta} \int_{0}^{\pi}\left|p_{k_{2}}\left(t_{2}\right) b_{n}(t)\right| d t+O(1) \int_{\delta}^{\pi} \int_{0}^{\pi}\left|b_{n}(t)\right| d t \\
& \leqslant \epsilon / 2+o(1)
\end{aligned}
$$

by (4.6).

It will be convenient to rewrite conditions (4.5) and (4.7) in sequence-tosequence form. Let $A=\left(a_{n v}\right)$ be a "multi-matrix"; that is, a matrix where the entries are indexed by the vectors $n$ and $\nu$ with the properties $a_{n \nu} \geqslant 0$, $a_{n \nu} \rightarrow 0(n \rightarrow \infty, \nu$ fixed $)$ and 


$$
\sum_{\nu \geq 0} a_{n \nu} \rightarrow 1 \quad(n \rightarrow \infty)
$$

$$
\sum_{\nu_{1}>k_{1}, \nu_{2}<k_{2}} a_{n \nu} \rightarrow 0 \quad(n \rightarrow \infty) \text { for } k=\left(k_{1}, k_{2}\right) \text { fixed, }
$$

$$
\sum_{\nu_{1}<k_{1}, \nu_{2}>k_{2}} a_{n \nu} \rightarrow 0 \quad(n \rightarrow \infty) \text { for } k \text { fixed. }
$$

When we feel it necessary we will write $a_{n \nu}=a_{n\left(\nu_{1}, \nu_{2}\right)}$.

A sequence $(S)=\left\{S_{n}\right\}$ is said to be convergent to $S$ if $\left|S_{n}\right| \leqslant M$ for all $n$ and $S_{n} \rightarrow S$ as $n \rightarrow \infty$. In this case, the above conditions on $A$ are necessary and sufficient for $A$ to be a regular sequence-to-sequence transformation. As a remark, we point out that for $n=\left(n_{1}, n_{2}\right)$ there are sequences for which $S_{n} \rightarrow S$ as $n \rightarrow \infty$ and $S_{n} \neq O(1)$. Furthermore, there is such a sequence such that $S_{n} \rightarrow 0$ as $n \rightarrow \infty$ but the $C_{1}$ mean of $(S)$ tends to $\infty$. Thus the boundedness condition on $(S)$ cannot be dispensed with.

Associated with $A$ is a series-to-sequence method $B=\left(b_{n v}\right)$ defined by

$$
b_{n \nu}=\sum_{\mu>\nu} a_{n \mu} \quad(n, \nu \geqslant 0 \text { are vectors })
$$

From the conditions on $A$ we see that

$$
\begin{array}{ll}
b_{n \nu} \rightarrow 1 & (n \rightarrow \infty, \nu \text { fixed }), \\
b_{n \nu} \rightarrow 0 & \left(n \text { fixed, } \nu_{1} \rightarrow \infty \text { or } v_{2} \rightarrow \infty\right)
\end{array}
$$

and if $\nu \geqslant \nu^{\prime}$ then $b_{n \nu} \leqslant b_{n \nu^{\prime}}$.

Suppose that $B$ satisfies condition (4.5). By writing $b_{n \nu}$ as the sum above and changing the order of summation (4.5) becomes

$$
\left|\sum_{m_{1}=1}^{k_{1}} \sum_{m_{2}=1}^{k_{2}} \frac{1}{m_{1} m_{2}} \sum_{\nu_{1}=k_{1}-m_{1}}^{k_{1}+m_{1}-1} \sum_{\nu_{2}=k_{2}-m_{2}}^{k_{2}+m_{2}-1} a_{n \nu}\right| \leqslant M .
$$

Similarly (4.7) becomes

(4.10a) $\lim _{n \rightarrow \infty} \sup _{k_{2}}\left|\sum_{m_{1}=1}^{k_{1}} \sum_{m_{2}=1}^{k_{2}} \frac{1}{m_{1} m_{2}} \sum_{\nu_{1}=k_{1}-m_{1}}^{k_{1}+m_{1}-1} \sum_{\nu_{2}=k_{2}-m_{2}}^{k_{2}+m_{2}-1} a_{n \nu}\right|=0$

for each $k_{1}$ and 
(4.10b) $\lim _{n \rightarrow \infty} \sup _{k_{1}}\left|\sum_{m_{1}=1}^{k_{1}} \sum_{m_{2}=1}^{k_{2}} \frac{1}{m_{1} m_{2}} \sum_{\nu_{1}=k_{1}-m_{1}}^{k_{1}+m_{1}-1} \sum_{\nu_{2}=k_{2}-m_{2}}^{k_{2}+m_{2}-1} a_{n \nu}\right|=0$ for each $k_{2}$.

5. Convergence theorems. In the next two sections, we prove two convergence results. The first of these shows that the method $L_{1}^{*}$ is Fourier-effective at Lebesgue points for which the maximal function is bounded. The second is that the method $L_{\alpha}^{*}$ is restrictedly Fourier-effective almost everywhere provided $\alpha>1$. For such $\alpha$, the method $L_{\alpha}^{*}$ is weaker than all two-dimensional Cesàro methods but still stronger than $L_{1}^{*}[4$, p. 257].

THEOREM 5.1. Let $t=0$ be a Lebesgue point for $\phi_{x}(t)$ at which the maximal function of $\phi_{x}(t)$ is bounded. Then $\sigma_{n, m}^{1}(x, f)=o(1)$ as $n \rightarrow \infty$ uniformly in $m, 0 \leqslant m \leqslant n$.

In the proof of this theorem, it will be convenient to use

$$
\mu_{x}(h)=\frac{1}{h_{1} h_{2}} \int_{0}^{h_{1}} \int_{0}^{h_{2}}\left|\phi_{x}(t)-f(x)\right| d t .
$$

Since $\mu_{x}(h) \rightarrow 0$ as $h \rightarrow 0$ and $M \phi_{x}(0) \leqslant M<\infty$ it follows that $\left|\mu_{x}(h)\right| \leqslant$ $M<\infty$.

Let $\omega$ be a nonnegative function of a single variable in $L^{1}[0, \pi]$. By using an integration by parts argument for each of the following integrals, one can show

$$
\begin{aligned}
\frac{1}{\epsilon} \int_{0}^{\epsilon} \omega(t) d t & +\int_{\epsilon}^{\delta} \omega(t) \frac{d t}{t}+\delta \cdot \int_{\delta}^{\pi} \omega(t) \frac{d t}{t^{2}} \\
& \leqslant \frac{\delta}{\pi} \rho(\pi)+\int_{\epsilon}^{\delta} \rho(t) \frac{d t}{t}+2 \delta \int_{\delta}^{\pi} \rho(t) \frac{d t}{t^{2}}
\end{aligned}
$$

where $\rho(t)=(1 / t) \int_{0}^{t} \omega(u) d u$. We will use (5.1) successively in the integrals appearing below.

We have as a consequence of (2.3), (2.4) and (2.5) with

$$
\epsilon_{i}=\left(n_{i}+1+m_{i}\right)^{-1}, \quad \delta_{i}=\left(n_{i}+1-m_{i}\right)^{-1} \text { for } i=1,2,
$$

and

$$
g(t)=\left(1+\log 1 /\left(1-t_{1}\right)\right)\left(1+\log 1 /\left(1-t_{2}\right)\right)
$$

that 


$$
\begin{gathered}
\left|g\left(\frac{m}{n+1}\right) \sigma_{n, m}^{1}(x, f)\right| \leqslant C \int_{0}^{\pi} \int_{0}^{\pi}\left|K_{n_{1}, m_{1}}\left(t_{1}\right) K_{n_{2}, m_{2}}\left(t_{2}\right)\left(\phi_{x}\left(t_{1}, t_{2}\right)-f(x)\right)\right| d t_{1} d t_{2} \\
=C \int_{0}^{\pi}\left|K_{n_{2}, m_{2}}\left(t_{2}\right)\right|\left\{\int_{0}^{\pi}\left|K_{n_{1}, m_{1}}\left(t_{1}\right)\right| \cdot\left|\phi_{x}\left(t_{1}, t_{2}\right)-f(x)\right| d t_{1}\right\} d t_{2} \\
\leqslant C \int_{0}^{\pi}\left|K_{n_{2}, m_{2}}\left(t_{2}\right)\right|\left\{\frac{1}{\epsilon_{1}} \int_{0}^{\epsilon_{1}} \omega\left(t_{1}, t_{2}\right) d t_{1}+\int_{\epsilon_{1}}^{\delta_{1}} \omega\left(t_{1}, t_{2}\right) \frac{d t_{1}}{t_{1}}\right. \\
\left.+\delta_{1} \int_{\delta_{1}}^{\pi} \omega\left(t_{1}, t_{2}\right) \frac{d t_{1}}{t_{1}^{2}}\right\} d t_{2}
\end{gathered}
$$

where $\omega\left(t_{1}, t_{2}\right)=\left|\phi_{x}\left(t_{1}, t_{2}\right)-f(x)\right|$ is integrable in $t_{1}$ for almost every $t_{2}$. One now applies (5.1) to the inner integrals and obtains a summation of three terms. In each of these, the estimates (2.3), (2.4) and (2.5) are applied again for variable $t_{2}$. One finally obtains that $\lg (m /(n+1)) \sigma_{n, m}^{1}\left(x, f^{\prime}\right) \mid$ is dominated by a constant times

$$
\begin{aligned}
\delta_{1} \delta_{2} \mu(\pi, \pi) & +2 \delta_{1} \delta_{2} \int_{\delta_{2}}^{\pi} \mu\left(\pi, t_{2}\right) \frac{d t_{2}}{t_{2}}+\delta_{1} \int_{\epsilon_{2}}^{\delta_{2}} \mu\left(\pi, t_{2}\right) \frac{d t_{2}}{t_{2}} \\
& +2 \delta_{1} \delta_{2} \int_{\delta_{1}}^{\pi} \mu\left(t_{1}, \pi\right) \frac{d t_{1}}{t_{1}^{2}}+4 \delta_{1} \delta_{2} \int_{\delta_{1}}^{\pi} \int_{\delta_{2}}^{\pi} \mu\left(t_{1}, t_{2}\right) \frac{d t_{1}}{t_{1}^{2}} \frac{d t_{2}}{t_{2}^{2}} \\
& +2 \delta_{1} \int_{\delta_{1}}^{\pi} \int_{\epsilon_{2}}^{\delta_{2}} \mu\left(t_{1}, t_{2}\right) \frac{d t_{1}}{t_{1}^{2}} \frac{d t_{2}}{t_{2}^{2}}+\delta_{2} \int_{\epsilon_{1}}^{\delta_{1}} \mu\left(t_{1}, \pi\right) \frac{d t_{1}}{t_{1}} \\
& +2 \delta_{2} \int_{\epsilon_{1}}^{\delta_{1}} \int_{\delta_{2}}^{\pi} \mu\left(t_{1}, t_{2}\right) \frac{d t_{1}}{t_{1}} \frac{d t_{2}}{t_{2}^{2}}+\int_{\epsilon_{1}}^{\delta_{1}} \int_{\epsilon_{2}}^{\delta_{2}} \mu\left(t_{1}, t_{2}\right) \frac{d t_{1}}{t_{1}} \frac{d t_{2}}{t_{2}}
\end{aligned}
$$

It is tedious to check all cases at this point. Instead, we pick terms from the above, which we feel are exemplary enough, and show they are $o(g(m /(n+1)))$ as $n \rightarrow \infty$ uniformly in $m, 0 \leqslant m \leqslant n$. We begin with

$$
I\left(\delta_{1}, \delta_{2}\right)=\delta_{1} \delta_{2} \int_{\delta_{1}}^{\pi} \int_{\delta_{2}}^{\pi} \mu\left(t_{1}, t_{2}\right) \frac{d t_{1}}{t_{1}^{2}} \frac{d t_{2}}{t_{2}^{2}} .
$$

First assume $\epsilon>0$ is given and that $\delta_{1}$ and $\delta_{2}$ tend to zero. Choose $\delta>0$ such that, for $0<t_{1}<\delta$ and $0<t_{2}<\delta$, we have (from the hypothesis) that $\mu\left(t_{1}, t_{2}\right)<\epsilon$. We break $I\left(\delta_{1}, \delta_{2}\right)$ into four integrals. These four are below with estimates. 


$$
\begin{aligned}
& I_{1}=\delta_{1} \delta_{2} \int_{\delta_{1}}^{\delta} \int_{\delta_{2}}^{\delta} \mu\left(t_{1}, t_{2}\right) \frac{d t_{1}}{t_{1}^{2}} \frac{d t_{2}}{t_{2}^{2}} \leqslant \epsilon \delta_{1} \delta_{2} \int_{\delta_{1}}^{\infty} \frac{d t_{1}}{t_{1}^{2}} \int_{\delta_{2}}^{\infty} \frac{d t_{2}}{t_{2}^{2}}=\epsilon . \\
& I_{2}=\delta_{1} \delta_{2} \int_{\delta}^{\pi} \int_{\delta_{2}}^{\delta} \mu\left(t_{1}, t_{2}\right) \frac{d t_{1}}{t_{1}^{2}} \frac{d t_{2}}{t_{2}^{2}} \leqslant M\left(\frac{\delta_{1}}{\delta}\right) \rightarrow 0 \text { as } \delta_{1} \rightarrow 0 . \\
& I_{3}=\delta_{1} \delta_{2} \int_{\delta}^{\pi} \int_{\delta}^{\pi} \mu\left(t_{1}, t_{2}\right) \frac{d t_{1}}{t_{1}^{2}} \frac{d t_{2}}{t_{2}^{2}} \leqslant M\left(\frac{\delta_{1} \delta_{2}}{\delta^{2}}\right) \rightarrow 0 \text { as } \delta_{1}, \delta_{2} \rightarrow 0 . \\
& I_{4}=\delta_{1} \delta_{2} \int_{\delta_{1}}^{\delta} \int_{\delta}^{\pi} \mu\left(t_{1}, t_{2}\right) \frac{d t_{1}}{t_{1}^{2}} \frac{d t_{2}}{t_{2}^{2}} \leqslant M\left(\frac{\delta_{2}}{\delta}\right) \rightarrow 0 \quad \text { as } \delta_{2} \rightarrow 0 .
\end{aligned}
$$

Next assume that $\delta_{1}$ or $\delta_{2}$ does not tend to zero; for example, assume $\delta_{1}$ remains bounded away from zero. Then

$$
g\left(\frac{m}{n+1}\right)=\left(1+\log \frac{n_{1}+1}{n_{1}-m_{1}+1}\right)\left(1+\log \frac{n_{2}+1}{n_{2}-m_{2}+1}\right)
$$

and $\delta_{1}$ bounded away from zero implies the first factor in $g(m /(n+1))$ tends to infinity as $n_{1} \rightarrow \infty$. However, $I\left(\delta_{1}, \delta_{2}\right) \leqslant M$. By using these remarks and some of the estimates in integrals $I_{1}, I_{2}, I_{3}$ and $I_{4}$ one sees $I\left(\delta_{1}, \delta_{2}\right)=$ $o(g(m /(n+1)))$ as $n \rightarrow \infty$ uniformly in $m, 0<m \leqslant n$ for this case. Other cases are similarly handled.

We consider one other example from the integrals in (5.2); namely,

$$
J=\int_{\epsilon_{1}}^{\delta_{1}} \int_{\epsilon_{2}}^{\delta_{2}} \frac{\mu\left(t_{1}, t_{2}\right)}{t_{1} t_{2}} d t_{1} d t_{2}
$$

If both $\delta_{1}$ and $\delta_{2}$ tend to zero then

$$
J=o(1) \int_{\epsilon_{1}}^{\delta_{1}} \int_{\epsilon_{2}}^{\delta_{2}} \frac{d t_{1} d t_{2}}{t_{1} t_{2}}=o\left(g\left(\frac{m}{n+1}\right)\right) .
$$

The case that $\delta_{1}$ and $\delta_{2}$ do not both tend to zero is handled in a manner similar to that for $I\left(\delta_{1}, \delta_{2}\right)$.

For future purposes, we remark that it can be easily seen from (5.2) that there exists a constant $C$ depending only on $M$, the bound for the maximal function, such that $\left|\sigma_{n, m}^{1}(x, f)\right| \leqslant C$ for all $n$ and $m$ with $0 \leqslant m \leqslant n$.

6. Convergence theorems (continued). In this section, we prove

THEOREM 6.1. Let $f \in L[0,2 \pi]^{2}$ and $\alpha>1$. Then for almost every $x, o_{n, m}^{\alpha}(x, f)$ tends to zero as $n$ tends restrictedly to infinity, uniformly in $m, 0 \leqslant m \leqslant n$. 
One should note that restricted summability implies the usual restrictedness relationships between $n_{1}$ and $n_{2}$ as well as a similar one for $m_{1}$ and $m_{2}$ while the converse is not true.

We will prove this convergence theorem by obtaining a maximal inequality. Let $\sigma^{*}(x, f)=\sup \left\{\left|\sigma_{n, m}^{\alpha}(x, f)\right|: 0 \leqslant m \leqslant n\right.$, restricted $\}$. This maximal function depends on the constant of restrictedness, $c$, but we suppress this fact. We will show there is a constant $C$ independent of $f \in L$ and $\lambda>0$ such that

$$
\left|\left\{\sigma^{*}(x, f)>\lambda\right\}\right| \leqslant C\|f\|_{1} / \lambda .
$$

In our proof, we obtain $C=C(\alpha)$ so that $C(\alpha) \rightarrow \infty$ as $\alpha \rightarrow 1$. It is clear that $\sigma_{n, m}^{\alpha}(x, f)$ is a linear operator on $f$ for $x$ fixed. We have

$$
\left[\varlimsup_{n \rightarrow \infty} \sigma_{n, m}^{\alpha}(x, f)-\varliminf_{n \rightarrow \infty} \sigma_{n, m}^{\alpha}(x, f)\right] \leqslant 2 \sigma^{*}(x, f)
$$

where the upper and lower limits are in the restricted sense. From Theorem 5.1, we know that $\varlimsup \sigma_{n, m}^{\alpha}(x, h)=\varliminf \sigma_{n, m}^{\alpha}(x, h)$ for a continuous function $h$. For an $\epsilon>0$ given we may write $f=g+h$ where $h$ is a continuous function and $\|g\|_{1}<\epsilon$. Let

$$
\begin{aligned}
A_{k} & =\left\{x: \varlimsup \overline{\lim } \sigma_{n, m}^{\alpha}(x, f)-\varliminf \sigma_{n, m}^{\alpha}(x, f)>1 / k\right\} \\
& =\left\{x: \varlimsup \overline{\lim } \sigma_{n, m}^{\alpha}(x, g)-\underline{\lim } \sigma_{n, m}^{\alpha}(x, g)>1 / k\right\} \\
& \subset\left\{x: 2 \sigma^{*}(x, g)>1 / k\right\} .
\end{aligned}
$$

It is easily seen that the set for which convergence does not hold is contained in $\bigcup_{k=1}^{\infty} A_{k}$. An application of (6.1) shows that $\left|A_{k}\right| \leqslant 2 k C \epsilon$. Since $\epsilon$ is arbitrary this shows $\left|A_{k}\right|=0$ and hence Theorem 6.1 follows from (6.1).

To obtain (6.1) we need

Lemma (6.2) (E. M. Stein AND N. J. Weiss [7, p. 37]). Let $g_{i}$ be positive functions and suppose that, for each $i=1,2, \cdots,\left|\left\{g_{i}>\lambda\right\}\right| \leqslant 1 / \lambda$. Suppose $c_{i}>0$ for $i=1,2, \cdots$ and $\Sigma c_{i}=1$. Then

$$
\left\{\sum c_{i} g_{i}>\lambda\right\} \leqslant 2(2+K) / \lambda \text { where } K=\sum_{i=1}^{\infty} c_{i} \log \left(1 / c_{i}\right) \text {. }
$$

We will also need

Lemma (6.3) [8, Vol. II, p. 310]. Let $h_{i}(t)$ be positive functions and strictly monotonic decreasing to zero as $t \rightarrow 0$, for $i=1,2$. Let

$$
f^{*}(x)=\sup \left\{\frac{1}{4 h_{1}(t) h_{2}(t)} \int_{-h_{1}(t)}^{h_{1}(t)} \int_{-h_{2}(t)}^{h_{2}(t)}|f(y)| d y: t>0\right\} .
$$


Then there exists a constant $C$ such that, for $f \in L, \quad\left|\left\{f^{*}(x)>\lambda\right\}\right| \leqslant C\|f\|_{1} / \lambda$ where $C$ is independent of $f, \lambda>0$, and $h_{i}, i=1,2$.

If we put

$$
\rho_{n, m}(x, f)=\frac{1}{\pi^{2} g(m /(n+1))} \int_{0}^{\pi} \int_{0}^{\pi}\left|f(x+t) \bar{K}_{n, m}(t)\right| d t,
$$

then we have $\left|\sigma_{n . m}^{\alpha}(x, f)\right| \leqslant \rho_{n, m}(x, f)+|f(x)|$ and (6.1) will follow from $\rho^{*}(x, f)=\sup \left\{\left|\rho_{n, m}(x, f)\right|: n, m\right.$ restricted $\left.\}\right)$

$$
\left|\left\{\rho^{*}(x, f)>\lambda\right\}\right| \leqslant C^{\prime}\|f\|_{1} \lambda \text {. }
$$

To further simplify calculation, we only prove the theorem for the special case $n_{1}=n_{2}$ and $m_{1}=m_{2}$. With the notation $\epsilon=\left(n_{1}+m_{1}+1\right)^{-1}, \delta=$ $\left(n_{1}-m_{1}+1\right)^{-1}$, and $|f|=\left|f\left(x_{1}+t_{1}, x_{2}+t_{2}\right)\right|$ and the estimates of (2.3), (2.4) and (2.5) we have. $\rho_{n, m}(x, f)$ is majorized by $C_{g}^{-1}(m /(n+1))$ times

$$
\sum_{i, j=1}^{3} I_{i j}=\frac{1}{\epsilon^{2}} \int_{0}^{\epsilon} \int_{0}^{\epsilon}|f| d t_{1} d t_{2}+\frac{1}{\epsilon} \int_{0}^{\epsilon} \int_{\epsilon}^{\delta}|f| d t_{1} \frac{d t_{2}}{t_{2}}+\frac{\delta}{\epsilon} \int_{0}^{\epsilon} \int_{\delta}^{\pi}|f| d t_{1} \frac{d t_{2}}{t_{2}^{2}}
$$

$$
\begin{aligned}
& +\frac{1}{\epsilon} \int_{\epsilon}^{\delta} \int_{0}^{\epsilon}|f| \frac{d t_{1}}{t_{1}} d t_{2}+\int_{\epsilon}^{\delta} \int_{\epsilon}^{\delta}|f| \frac{d t_{1}}{t_{1}} \frac{d t_{2}}{t_{2}}+\delta \int_{\epsilon}^{\delta} \int_{\delta}^{\pi}|f| \frac{d t_{1}}{t_{1}} \frac{d t_{2}}{t_{2}^{2}} \\
& +\frac{\delta}{\epsilon} \int_{\delta}^{\pi} \int_{0}^{\epsilon}|f| \frac{d t_{1}}{t_{1}^{2}} d t_{2}+\delta \int_{\delta}^{\pi} \int_{\epsilon}^{\delta}|f| \frac{d t_{1}}{t_{1}^{2}} \frac{d t_{2}}{t_{2}}+\delta^{2} \int_{\delta}^{\pi} \int_{\delta}^{\pi}|f| \frac{d t_{1}}{t_{1}^{2}} \frac{d t_{2}}{t_{2}^{2}}
\end{aligned}
$$

where the sum is arranged in an array to suggest the indexing for the summation.

We will use Lemma (6.3) with $h_{1}(t)=2^{t} t$, and $h_{2}(t)=2^{i} t$. The corresponding maximal functions are

$$
f_{i, j}^{*}(x)=\sup _{\epsilon>0} \frac{1}{\epsilon^{2} 2^{i} 2^{j}} \int_{-\epsilon 2^{i}}^{\epsilon 2^{i}} \int_{-\epsilon 2^{j}}^{\epsilon 2^{j}}\left|f\left(x_{1}+t_{1}, x_{2}+t_{2}\right)\right| d t_{1} d t_{2} .
$$

We will also use the following sums of such maximal functions

$$
\begin{gathered}
F_{11}(x)=f_{0,0}^{*}(x), \quad F_{12}(x)=\sum_{j=1}^{\infty} j^{-\alpha} f_{0, j}^{*}(x), \quad F_{13}(x)=\sum_{j=1}^{\infty} 2^{-j} f_{0, j}^{*}(x), \\
F_{21}(x)=\sum_{i=1}^{\infty} i^{-\alpha} f_{i, 0}^{*}(x), \quad F_{22}(x)=\sum_{i, j=1}^{\infty}(i j)^{-\alpha} f_{i, j}^{*}(x), \quad F_{23}(x)=\sum_{i, j=1}^{\infty} i^{-\alpha} 2^{-j} f_{i, j}^{*}(x),
\end{gathered}
$$




$$
F_{31}(x)=\sum_{i=1}^{\infty} 2^{-i} f_{i, 0}^{*}(x), F_{32}(x)=\sum_{i, j=1}^{\infty} 2^{-i} j^{-\alpha} f_{i, j}^{*}(x), F_{33}(x)=\sum_{i, j=1}^{\infty} 2^{-(i+j)} f_{i, j}^{*}(x) .
$$

To apply Lemma 6.2, we agree to let the maximal functions in any one of the above sums be reindexed and denoted by $f_{i}^{*}(x)$. It follows from Lemma 6.3 that each $f_{i}^{*}$ is subject to a weak type inequality with a weak type constant $C_{0}$ which is the same for all $f_{i}^{*}(x)$. Furthermore, we allow the numerical factors to be reindexed and denoted by $a_{i}$. Then any one of the nine expressions becomes an expression of the form $\Sigma_{i=1}^{\infty} a_{i} f_{i}^{*}(x)=\bar{f}(x)$.

We now show that $\bar{f}$ satisfies a maximal inequality of type (6.1 $)$. Let $g_{i}=f_{i}^{*}(x) / C\|f\|, \quad a=\Sigma_{i=1}^{\infty} a_{i}, \quad c_{i}=a_{i} / a$ for $i=1,2, \cdots$ where $C$ is the universal weak type constant of Lemma 6.3. With these choices, the $g_{i}$ and $c_{i}$ satisfy the hypotheses of Lemma 6.2 . Hence

$$
\left|\left\{\sum c_{i} g_{i}>\lambda\right\}\right| \leqslant 2(K+2) / \lambda \text { where } K=(1 / a) \sum a_{i} \ln \left(a / a_{i}\right) .
$$

It follows that

$$
\left|\left\{\sum a_{i} f_{i}^{*}>\lambda\right\}\right|=\left|\left\{\sum \frac{a_{i}}{a} \frac{f_{i}^{*}}{C\|f\|}>\frac{\lambda}{a C\|f\|}\right\}\right| \leqslant \frac{2 a C}{\lambda}(K+2)\|f\| .
$$

With this remark, it remains to show that each of the expressions on the right of (6.2) satisfies an inequality of the type

$$
I_{i j}(x) \leqslant C F_{. i j}^{*}(x) \text {. }
$$

Because of the tedious nature of this task we again choose to give a proof in two exemplary cases.

We do this first for $I_{22}$. Let $L$ be the smallest integer such that $2^{L} \geqslant$ $\delta / \epsilon$. In this case, it follows that $L^{2 \alpha} g^{-1}(m /(n+1)) \leqslant C<\infty$ for all $n$ and $m$, $0 \leqslant m \leqslant n$. Then

$$
\begin{aligned}
I_{22} & =\frac{1}{g(m /(n+1))} \int_{\epsilon}^{\delta} \int_{\epsilon}^{\delta}|f| \frac{d t_{1}}{t_{1}} \frac{d t_{2}}{t_{2}} \\
& \leqslant \frac{1}{g(m /(n+1))} \sum_{i, j=1}^{L} \int_{2}^{2^{i} \epsilon} \int_{i^{i} \epsilon^{2} \int^{j}{ }^{j} \epsilon \epsilon}|f| \frac{d t_{1}}{t_{1}} \frac{d t_{2}}{t_{2}} \\
& \leqslant \frac{4 L^{2 \alpha}}{g(m /(n+1))} \sum_{i, j=1}^{L}(i j)^{-\alpha} f_{i, j}^{*}(x) \leqslant C F_{22}^{*}(x) .
\end{aligned}
$$

We consider $I_{33}$ as our second example. This time, let $L$ be the smallest integer for which $2^{L} \delta \geqslant \pi$. Then 


$$
\begin{aligned}
I_{33}(x) & =\frac{\delta^{2}}{g(m /(n+1))} \int_{\delta}^{\pi} \int_{\delta}^{\pi}|f| \frac{d t_{1}}{t_{1}^{2}} \frac{d t_{2}}{t_{2}^{2}} \\
& \leqslant \frac{\delta^{2}}{g(m /(n+1))} \sum_{i, j=1}^{L} \int_{2^{i-1} \delta}^{2^{i} \delta} \int_{2^{j-1} \delta}^{2^{j} \delta}|f| \frac{d t_{1}}{t_{1}^{2}} \frac{d t_{2}}{t_{2}^{2}} \\
& \leqslant C \sum_{i, j=1}^{L} 4 \cdot 2^{-i} 2^{-i} f_{i, j}^{*}(x) \leqslant C F_{33}^{*}(x) .
\end{aligned}
$$

Similar calculations give the remaining inequalities of (6.3). A finite "sum" of weak type inequalities gives rise to a weak type inequality. In our case (6.1) follows from the weak type inequalities for the $F_{i j}^{*}(x)$.

A modification of the above argument gives the full restricted version. To see a method for doing this one could follow the argument given for the $C_{1}$ case, as done in [8, Vol. II, p. 311]. One should alsc compare Theorem 6.1 with the result given there.

7. Characterization of monotone effective methods. In this section we will prove

THEOREM 7.1. A two-dimensional monotone summability method $A$ is Fourier-effective if and only if $A \supseteq L_{1}^{*}$.

In what follows we will see that for monotone methods $F_{C}$-effectiveness and $F_{L}$-effectiveness are identical, thus we may speak of effectiveness without qualification. The definition of monotone will be given shortly. Theorem 7.1 will follow from Theorem 7.2.

In $\S 5$ we proved that $L_{1}^{*}$ is $F_{L}$ (and hence $F_{C}$ ) effective. It follows trivially that if $A \supseteq L_{1}^{*}$ then $A$ is effective. So we need only prove that if $A$ is monotone and effective, then $A \supseteq L_{1}^{*}$.

In this direction, we have already shown that if a nonnegative regular method $A$ is effective, then (4.9) holds. If, in addition, we have (4.6), then (4.10) holds. It is easily seen that (4.9) implies (see [3, p. 248])

$$
\sum_{\nu_{1}=0}^{k_{1}-1} \sum_{\nu_{2}=0}^{k_{2}-1} a_{n \nu}\left(1+\log \frac{k_{1}}{k_{1}-\nu_{1}}\right)\left(1+\log \frac{k_{2}}{k_{2}-\nu_{2}}\right)=O(1)
$$

$$
\sum_{\nu_{1}=0}^{k_{1}-1} \sum_{\nu_{2}=k_{2}}^{\infty} a_{n \nu}\left(1+\log \frac{k_{1}}{k_{1}-\nu_{1}}\right)\left(1+\log \frac{\nu_{2}+1}{\nu_{2}+1-k_{2}}\right)=O(1)
$$


(7.1c) $\sum_{\nu_{1}=k_{1}}^{\infty} \sum_{\nu_{2}=0}^{k_{2}-1} a_{n \nu}\left(1+\log \frac{\nu_{1}+1}{\nu_{1}+1-k_{1}}\right)\left(1+\log \frac{k_{2}}{k_{2}-\nu_{2}}\right)=O(1)$,

(7.1d) $\sum_{\nu_{1}=k_{1}}^{\infty} \sum_{\nu_{2}=k_{2}}^{\infty} a_{n \dot{\nu}}\left(1+\log \frac{\nu_{1}+1}{\nu_{1}+1-k_{1}}\right)\left(1+\log \frac{\nu_{2}+1}{\nu_{1}+1-k_{2}}\right)=O(1)$

for $n \geqslant 0, k \geqslant 1$ and that (410a) implies

$$
\sup _{k_{2}} \sum_{\nu_{1}=0}^{k_{1}-1} \sum_{\nu_{2}=0}^{k_{2}-1} a_{n \nu}\left(1+\log \frac{k_{1}}{k_{1}-\nu_{1}}\right)\left(1+\log \frac{k_{2}}{k_{2}-\nu_{2}}\right)=o(1)
$$

(7.2b) $\sup _{k} \sum_{\nu_{1}=0}^{k_{1}-1} \sum_{\nu_{2}=k_{2}}^{\infty} a_{n \nu}\left(1+\log \frac{k_{1}}{k_{1}-v_{1}}\right)\left(1+\log \frac{\nu_{2}+1}{\nu_{2}+1-k_{2}}\right)=o(1)$.

Interchanging indices 1 and 2 will give conditions we call (7.2c) and (7.2d) which follow from (4.10b).

A method $A$ is monotone if $A$ is regular, nonnegative and, with the following notation

$$
\begin{aligned}
& \Delta^{10} a_{n \nu}=a_{n\left(\nu_{1}, \nu_{2}\right)}-a_{n\left(\nu_{1}+1, \nu_{2}\right)}, \\
& \Delta^{01} a_{n \nu}=a_{n\left(\nu_{1}, \nu_{2}\right)}-a_{n\left(\nu_{1}, \nu_{2}+1\right)}, \\
& \Delta^{11} a_{n \nu}=a_{n\left(\nu_{1}, \nu_{2}\right)}-a_{n\left(\nu_{1}+1, \nu_{2}\right)}-a_{n\left(\nu_{1}, \nu_{2}+1\right)}+a_{n\left(\nu_{1}+1, \nu_{2}+1\right)}
\end{aligned}
$$

we have

$$
\begin{aligned}
\Delta^{10} a_{n \nu} & \leqslant 0 \text { if } 0 \leqslant v_{1}<v_{1}(n), \\
& \geqslant 0 \text { if } v_{1}(n) \leqslant v_{1}, \\
\Delta^{01} a_{n \nu} & \leqslant 0 \text { if } 0 \leqslant v_{2}<v_{2}(n), \\
& \geqslant 0 \text { if } v_{2}(n) \leqslant v_{2}, \\
\Delta^{11} a_{n \nu} & \geqslant 0 \text { if } 0 \leqslant \nu<\nu(n) \text { or } \nu \geqslant \nu(n), \\
& \leqslant 0 \text { otherwise, }
\end{aligned}
$$

where $\nu(n)$ is a function of $n$ specified for $A$. All methods $A$ formed by taking the product of two one-dimensional monotone methods are monotone; for 
example, all Cesàro methods with positive order are monotone.

Assuming $g$ is defined on $[0,1)^{2}$, we define $h(\nu ; k)=h\left(\nu_{1}, \nu_{2} ; k_{1}, k_{2}\right)$ by

$$
h(\nu ; k)= \begin{cases}g\left(\frac{\nu}{k}\right) ; & 0 \leqslant \nu<k, \\ g\left(\frac{\nu_{1}}{k_{1}}, \frac{k_{2}}{\nu_{2}+1}\right) ; & 0 \leqslant \nu_{1}<k_{1}, k_{2} \leqslant v_{2}, \\ g\left(\frac{k_{1}}{\nu_{1}+1}, \frac{\nu_{2}}{k_{2}}\right) ; & k_{1}<\nu_{1}, 0 \leqslant v_{2}<k_{2}, \\ g\left(\frac{k}{\nu+1}\right) ; & \nu \geqslant k,\end{cases}
$$

for all $\nu \geqslant 0$ and $k \geqslant 1$.

TheOREM 7.2. Suppose $g(t) \uparrow$ as $t \uparrow$ (using the order defined in \$2), $t \in[0,1)^{2}$, and $A$ is monotone. Suppose that

$$
\sum_{\nu \geq 0} a_{n \nu} h(\nu ; \nu(n)+1)=O(1)
$$$$
\lim _{n \rightarrow \infty} \sup _{k_{2}} \sum_{\nu_{2}=0}^{\infty} a_{n \nu} h(\nu ; k)=0 \text { for each } \nu_{1}, k_{1} \text {, }
$$

$$
\lim _{n \rightarrow \infty} \sup _{k_{1}} \sum_{\nu_{1}=0}^{\infty} a_{n \nu} h(\nu ; k)=0 \text { for each } \nu_{2}, k_{2},
$$

then $A \supseteq[g]$.

Note that for

$$
g\left(\nu_{1}, v_{2}\right)=\left(1+\log \frac{1}{1-v_{1}}\right)\left(1+\log \frac{1}{1-v_{2}}\right) \text { and } k=v(n)+1 \text {, }
$$

(7.4) is (7.1), and (7.5) follows from (7.2). Furthermore (7.6) and (7.7) follow from the definition of $g$. For example, if $\nu<\nu(n)$ then 


$$
\begin{aligned}
\left|\Delta^{11} h(\nu ; \nu(n)+1)\right| & =\left|\Delta^{11} g\left(\frac{\nu}{\nu(n)+1}\right)\right| \\
& =\left|\log \frac{\nu_{1}(n)-\nu_{1}}{\nu_{1}(n)+1-\nu_{1}} \log \frac{\nu_{2}(n)-\nu_{2}}{\nu_{2}(n)+1-\nu_{2}}\right| \\
& =\int_{\nu_{1}(n)-\nu_{1}}^{\nu_{1}(n)+1-\nu_{1}} \frac{d t}{t} \int_{\nu_{2}(n)-\nu_{2}}^{\nu_{2}(n)+1-\nu_{2}} \frac{d t}{t} \leqslant \frac{1}{\nu_{1}(n)-\nu_{1}} \cdot \frac{1}{\nu_{2}(n)-\nu_{2}} .
\end{aligned}
$$

Thus, in order to show that Theorem 7.1 follows from Theorem 7.2, we need only prove

LEMmA 7.1. If $A$ is monotone and satisfies (7.1a), then $\int_{T_{\delta}}\left|b_{n}(t)\right| d t \rightarrow 0$ as $n \rightarrow \infty$.

Here, $T_{\delta}$ and $b_{n}$ are as in Theorem 4.1.

Proof. Since $A$ is nonnegative and regular we may apply summation by parts twice to obtain (compare $[3$, p. 239, formula just below (3.5)] and [8, Vol. I, p. 183, (1.7)])

$$
b_{n}(t)=\sum_{\nu>0}\left(\nu_{1}+1\right)\left(\nu_{2}+1\right) \Delta^{11} a_{n \nu} K_{\nu}(t)
$$

where $K_{\nu}^{\prime}(t)$ is the two-dimensional Fejér kernel. Fix $t_{1}$ and $t_{2}>0$. By a standard estimate on $K_{\nu}(t)$, we obtain

$$
\begin{aligned}
\left|b_{n}(t)\right| \leqslant \frac{C}{t_{1}^{2} t_{2}^{2}} \sum_{\nu>0}\left|\Delta^{11} a_{n \nu}\right| & \\
= & \frac{C}{t_{1}^{2} t_{2}^{2}}\left[\sum_{\nu=0}^{\nu(n)-1} \Delta^{11} a_{n \nu}-\sum_{\nu_{1}=0}^{\nu_{1}(n)-1} \sum_{\nu_{2}=\nu_{2}(n)}^{\infty} \Delta^{11} a_{n \nu}\right. \\
& \left.\quad-\sum_{\nu_{1}=\nu_{1}(n)}^{\infty} \sum_{\nu_{2}=0}^{\nu_{2}(n)-1} \Delta^{11} a_{n \nu}+\sum_{\nu=\nu(n)}^{\infty} \Delta^{11} a_{n \nu}\right]
\end{aligned}
$$

since $A$ is monotone. But each term in the brackets tends to zero as $n \rightarrow \infty$. Consider, for example, the first term. We have

$$
\sum_{\nu=0}^{\nu(n)-1} \Delta^{11} a_{n \nu}=a_{n 0}-a_{n\left(\nu_{1}(n) ; 0\right)}-a_{n\left(0, \nu_{2}(n)\right)}+a_{n \nu(n)} \text {, }
$$

and the regularity of $A$ implies that the first three terms on the right tend to zero. If either $v_{1}(n)$ or $v_{2}(n)$ is bounded, then $a_{n \nu(n)} \rightarrow 0$ as $n \rightarrow \infty$ 
also by the regularity of $A$. If $\nu_{1}(n)$ and $\nu_{2}(n)$ both tend to infinity as $n \rightarrow \infty$, then by (7.1a),

$$
a_{n v(n)}\left(1+\log \left(\nu_{1}(n)+1\right)\right)\left(1+\log \left(\nu_{2}(n)+1\right)\right)
$$

is bounded and it follows that $a_{n \nu(n)} \rightarrow 0$. That $a_{n \nu(n)} \rightarrow 0$ as $n \rightarrow \infty$ for a general $\nu(n)$ follows from these two special cases.

Therefore, $\lim _{n \rightarrow \infty}\left|b_{n}(t)\right|=0$ for each $t_{1}, t_{2}>0$. By (4.1), $\left|b_{n}\right|$ is essentially bounded on $T_{\delta}$ and an application of the bounded convergence theorem gives the result.

Proof of Theorem 7.2. Suppose $(t) \in[g]$; that is,

$$
\frac{1}{g(m /(n+1))} \frac{1}{*(n+1-m)} \sum_{m<\nu<n}\left(t_{\nu}-t\right)=o(1)
$$

as $n \rightarrow \infty$ uniformly for $0 \leqslant m \leqslant n$, where by $o(1)$ we will mean, throughout this section, a function which is bounded and tends to 0 as $n$ tends to infinity. One should compare this to the final remark of $\$ 5$. It will be convenient to extend the definition of $g$ to all of $[0, \infty)^{2}$ by

$$
g(x, y)= \begin{cases}0 & \text { if either } x=1 \text { or } y=1, \\ g(1 / x, y) & \text { if } x>1, y<1 \\ g(x, 1 / y) & \text { if } x<1, y>1 \\ g(1 / x, 1 / y) & \text { if } x>1, y>1\end{cases}
$$

Let $s_{\nu}=t_{\nu}-t$ for $\nu \geqslant 0$ and zero otherwise, $s_{m \cdot}=\Sigma_{\nu<m} s_{\nu}, \sigma_{m}=$ $\left({ }^{*}(m+1)\right)^{-1} S_{m}$ and define $S_{m}^{n}=S_{\nu}-S_{\nu_{1}, k_{2}}-S_{k_{1}, \nu_{2}}+S_{k}$. Thus

$$
S_{m}^{n}= \begin{cases}\sum_{m<\nu<n} s_{\nu} & (m<n), \\ \sum_{n<\nu<m} s_{\nu} & (m>n), \\ -\sum_{\nu_{1}=n_{1}+1}^{m_{1}} \sum_{\nu_{2}=m_{2}+1}^{n_{2}} s_{\nu} & \left(m_{1}>n_{1}, m_{2}<n_{2}\right), \\ -\sum_{\nu_{1}=m_{1}+1}^{n_{1}} \sum_{\nu_{2}=n_{2}+1}^{m_{2}} s_{\nu} & \left(m_{1}<n_{1}, m_{2}>n_{2}\right), \\ 0 & \text { otherwise. }\end{cases}
$$


Further, if we define

$$
\tau(m, n)= \begin{cases}S_{m}^{n} \frac{1}{\left.\right|^{*}(m-n) \mid g((m+1) /(n+1))} & \left(m_{1} \neq n_{1}, m_{2} \neq n_{2}\right), \\ 0 \quad \text { otherwise, } & \end{cases}
$$

and $j_{1}=\max \left(m_{1}, n_{1}\right), j_{2}=\max \left(m_{2}, n_{2}\right), i_{1}=\min \left(m_{1}, n_{1}\right), i_{2}=\min \left(m_{2}, n_{2}\right)$, then (7.8) can be rewritten as

$$
\sup _{-1<i<j} \tau(m, n)=o(1) \text { as } j \rightarrow \infty .
$$

Summing by parts gives

$$
\begin{aligned}
\sum_{0<\nu<m} a_{n \nu} s_{\nu}= & S_{m} a_{n, m+1}+\sum_{\nu_{1}=0}^{m_{1}} S_{\nu_{1}, m_{2}} \Delta^{10} a_{n\left(\nu_{1}, m_{2}+1\right)} \\
& +\sum_{\nu_{2}=0}^{m_{2}} S_{m_{1}, \nu_{2}} \Delta^{01} a_{n\left(m_{1}+1, \nu_{2}\right)}+\sum_{0<\nu<m} S_{\nu} \Delta^{11} a_{n \nu} .
\end{aligned}
$$

A short calculation shows that this formula can, for a given $k$, be expanded to

$$
\sum_{0<\nu<m} a_{n \nu} S_{\nu}=\left(S_{m}-S_{k}\right) a_{n, m+1}+\sum_{\nu_{1}=0}^{m_{1}}\left(S_{\nu_{1}, m_{2}}-S_{\nu_{1}, k_{2}}\right) \Delta^{10} a_{n\left(\nu_{1}, m_{2}+1\right)}
$$

$$
+\sum_{\nu_{2}=0}^{m_{2}}\left(S_{m_{1}, \nu_{2}}-S_{k_{1}, \nu_{2}}\right) \Delta^{01} a_{n\left(m_{1}+1, \nu_{2}\right)}
$$

$$
\begin{aligned}
& +\sum_{0<\nu<m} S_{k}^{\nu} \Delta^{11} a_{n \nu}+\sum_{\nu_{1}=0}^{m_{1}}\left(S_{\nu_{1}, k_{2}}-S_{k}\right) \Delta^{10} a_{n\left(\nu_{1}, 0\right)} \\
& +\sum_{\nu_{2}=0}^{m_{2}}\left(S_{k_{1}, \nu_{2}}-S_{k}\right) \Delta^{01} a_{n\left(0, \nu_{2}\right)}+S_{k} a_{n 0} .
\end{aligned}
$$

We first show that for fixed $n$ and $k$ the first three terms of (7.10) tend to 0 as $m \rightarrow \infty$. Because $A$ is monotone one can show that $\left(m_{1}+1\right)\left(m_{2}+1\right) a_{n, m+1}$ $\rightarrow 0$. Thus $S_{m} a_{n, m+1}=\left(m_{1}+1\right)\left(m_{2}+1\right) \sigma_{m} a_{n, m+1} \rightarrow 0$ as $m \rightarrow \infty$ and it follows that the first term of (7.10) tends to zero as $m$ tends to infinity.

Using (7.9) we have that the second term of (7.10) is, for large $m$, equal to 


$$
\begin{aligned}
\sum_{\nu_{1}=0}^{m_{1}} \tau\left(-1, k_{2} ; \nu_{1}, m_{2}\right)\left(\nu_{1}+1\right)\left|m_{2}-k_{2}\right| g\left(0, \frac{k_{2}+1}{m_{2}+1}\right) \mid \Delta^{10} a_{n\left(\nu_{1}, m_{2}+1\right) \mid} & {\left[\sum_{\nu_{1}=0}^{\nu_{1}(n)-1}\left(\nu_{1}+1\right)\left(a_{n\left(\nu_{1}+1, m_{2}+1\right)}-a_{n\left(\nu_{1}, m_{2}+1\right)}\right)\right.} \\
=O(1)\left(m_{2}+1\right) & \left.-\sum_{\nu_{1}=\nu_{1}(n)}^{m_{1}}\left(\nu_{1}+1\right)\left(a_{n\left(\nu_{1}+1, m_{2}+1\right)}-a_{n\left(\nu_{1}, m_{2}+1\right)}\right)\right] \\
=O(1)\left(m_{2}+1\right)\left[2 \nu_{1}(n) a_{n\left(\nu_{1}(n), m_{2}+1\right)}+\sum_{\nu_{1}=\nu_{1}(n)}^{m_{1}} a_{n\left(\nu_{1}, m_{2}+1\right)}\right. & \left.-\sum_{\nu_{1}=0}^{\nu_{1}(n)-1} a_{n\left(\nu_{1}, m_{2}+1\right)}-\left(m_{1}+1\right) a_{n, m+1}\right]
\end{aligned}
$$

and $\left(m_{2}+1\right)$ times each of the terms in brackets tends to zero as $m \rightarrow \infty$. Consider, for example, the second term. We have

$$
\sum_{\nu_{1}=\nu_{1}(n)}^{m_{1}} a_{n\left(\nu_{1}, \rho\right)} \leqslant \sum_{\nu_{1}=\nu_{1}(n)}^{\infty} a_{n\left(\nu_{1}, \rho\right)} \equiv V_{\rho}
$$

and $V_{\rho} \downarrow$ for all large $\rho$ because $A$ is monotone, and $\Sigma V_{\rho}$ exists since $A$ is regular. It follows that $\rho V_{\rho} \rightarrow 0$ as $\rho \rightarrow \infty$.

Similarly it can be shown that the third term of (7.10) tends to zero as $m \rightarrow \infty$.

We now have justified writing

$$
\begin{aligned}
\sum_{\nu>0} a_{n \nu} s_{\nu}= & \sum_{\nu>0} S_{k}^{\nu} \Delta^{11} a_{n \nu}+\sum_{\nu_{1}=0}^{\infty}\left(S_{\nu_{1} k_{2}}-S_{k}\right) \Delta^{10} a_{n\left(\nu_{1}, 0\right)} \\
& +\sum_{\nu_{2}=0}^{\infty}\left(S_{k_{1} \nu_{2}}-S_{k}\right) \Delta^{01} a_{n\left(0, \nu_{2}\right)}+S_{k} a_{n 0}
\end{aligned}
$$

and to complete the proof we must show that, for a choice of $k$ depending on $n$, each of these terms is $o(1)$ as $n \rightarrow \infty$. We will show how this follows from the conditions below.

$$
\sum_{\nu>0}\left|*(\nu-\nu(n)) g\left(\frac{\nu+1}{\nu(n)+1}\right) \Delta^{11} a_{n \nu}\right|=O(1)
$$


(7.13a) $\left(\nu_{2}(n)+1\right) \sum_{\nu_{1}=0}^{\infty}\left|\left(\nu_{1}-\nu_{1}(n)\right) g\left(\frac{\nu_{1}+1}{\nu_{1}(n)+1}, 0\right) \Delta^{10} a_{n\left(\nu_{1}, 0\right)}\right|=O(1)$,

(7.13b) $\left(\nu_{1}(n)+1\right) \sum_{\nu_{2}=0}^{\infty}\left|\left(\nu_{2}-v_{2}(n)\right) g\left(0, \frac{\nu_{2}+1}{v_{2}(n)+1}\right) \Delta^{01} a_{n\left(0, \nu_{2}\right)}\right|=O(1)$,

$$
*(\nu(n)+1) a_{n 0}=O(1)
$$

(7.15a) $\lim _{n \rightarrow \infty} \sum_{\nu_{1}=0}^{\infty}\left|\nu_{1}(n)-\nu_{1}\right| g\left(\frac{\nu_{1}+1}{\nu_{1}(n)+1}, 0\right)\left|\Delta^{11} a_{n \nu}\right|=0$ for each $\nu_{2}$,

(7.15b) $\lim _{n \rightarrow \infty} \sum_{\nu_{2}=0}^{\infty}\left|\nu_{2}(n)-\nu_{2}\right| g\left(0, \frac{\nu_{2}+1}{v_{2}(n)+1}\right)\left|\Delta^{11} a_{n \nu}\right|=0$ for each $v_{1}$.

We see this first for the case $\nu(n) \rightarrow \infty$. Let $k=\nu(n)$. Then the first term of (7.11) is majorized by

$$
\sum_{\nu>0}\left|\tau(\nu, \nu(n)) *(\nu-\nu(n)) g\left(\frac{\nu+1}{\nu(n)+1}\right) \Delta^{11} a_{n \nu}\right|
$$

which is $o(1)$ by (7.9) and (7.12). The second term of (7.11) is majorized by

$$
\left(\nu_{2}(n)+1\right) \sum_{\nu_{1}=0}^{\infty}\left|\gamma\left(\nu_{1},-1 ; \nu(n)\right)\left(\nu_{1}-\nu_{1}(n)\right) g\left(\frac{\nu_{1}+1}{\nu_{1}(n)+1}, 0\right) \Delta^{10} a_{n\left(\nu_{1}, 0\right)}\right|
$$

which is $o(1)$ by (7.9) and (7.13a). Similarly, the third term of (7.11) is $o(1)$ by conditions (7.9) and (7.13b). Finally, the last term of (7.11) is $o(1)$ because of (7.9) and (7.14).

Next suppose that $\nu_{1}(n) \rightarrow \infty$ while $\nu_{2}(n)=O(1)$, as $n \rightarrow \infty$. For this case let $k_{1}=v_{1}(n)$ and $k_{2}=-1$. Then $S_{k_{1},-1}=0$ in (7.11) and we obtain

(7.16) $\sum_{\nu>0} a_{n \nu} s_{\nu}=\sum_{\nu>0} S_{\left(\nu_{1}(n),-1\right)} \Delta^{11} a_{n \nu}+\sum_{\nu_{2}=0}^{\infty} S_{\left(\nu_{1}(n), \nu_{2}\right)^{\Delta}} a^{01} a_{n\left(0, \nu_{2}\right)}$

The second term of (7.16) is majorized by

$$
\left(\nu_{1}(n)+1\right) \sum_{\nu_{2}=0}^{\infty}\left(\nu_{2}+1\right)\left|\sigma_{\left(\nu_{1}(n), \nu_{2}\right)} \Delta^{01} a_{n\left(0, \nu_{2}\right)}\right|
$$


Choose $\epsilon>0$. Choose $M$ such that $\nu_{1}>M$ and $\nu_{2}>M$ implies $\sigma_{\nu}<\epsilon / 2 P$ where

$$
\begin{aligned}
& P= \sup _{n}\left(\nu_{1}(n)+1\right) \sum_{\nu_{2}=0}^{\infty}\left(\nu_{2}+1\right)\left|\Delta^{01} a_{n\left(0, \nu_{2}\right)}\right| \\
& \leqslant \sup _{n}\left(\nu_{1}(n)+1\right) \sum_{\nu_{2}=0}^{\infty}\left|\nu_{2}-\nu_{2}(n)\right| \cdot\left|\Delta^{01} a_{n\left(0, \nu_{2}\right)}\right| \\
& \quad+\sup _{n} \sum_{\nu_{1}=0}^{\nu_{1}(n)} \sum_{\nu_{2}=0}^{\infty}\left(\nu_{2}(n)+1\right)\left|\Delta^{01} a_{n\left(0, \nu_{2}\right)}\right|<\infty
\end{aligned}
$$

by (7.13b) and the regularity of $A$. Hence, if $\nu_{1}(n)>M$, then (7.17) is majorized by

$$
\begin{aligned}
\frac{\epsilon}{2}+\left(\nu_{1}(n)+1\right) \sum_{\nu_{2}=0}^{M}\left(\nu_{2}\right. & +1)\left|\sigma_{\left(\nu_{1}(n), \nu_{2}\right)} \Delta^{01} a_{n\left(0, \nu_{2}\right)}\right| \\
& \leqslant \frac{\epsilon}{2}+C \sum_{\nu_{1}=0}^{\nu_{1}(n)} \sum_{\nu_{2}=0}^{M}\left|\Delta^{01} a_{n \nu}\right|<\epsilon
\end{aligned}
$$

for all sufficiently large $n$, by the regularity and monotonicity of $A$.

The first term of (7.16) is majorized by

$$
\begin{aligned}
\sum_{\nu>0} \tau\left(\nu_{1}(n),\right. & -1 ; \nu)\left(\nu_{2}+1\right)\left|\nu_{1}(n)-\nu_{1}\right| g\left(\frac{\nu_{1}+1}{\nu_{1}(n)+1}, 0\right)\left|\Delta^{11} a_{n \nu}\right| \\
& <C \sum_{\nu_{2}=0}^{M} \sum_{\nu_{1}=0}^{\infty}\left|\nu_{1}(n)-\nu_{1}\right| g\left(\frac{\nu_{1}+1}{\nu_{1}(n)+1}, 0\right)\left|\Delta^{11} a_{n \nu}\right|+\frac{\epsilon}{2}<\epsilon
\end{aligned}
$$

for all sufficiently large $n$ by (7.9), (7.12) and (7.15a).

The case in which $\nu_{1}(n)=O(1)$ and $\nu_{2}(n) \rightarrow \infty$ as $n \rightarrow \infty$ is handled in a manner similar to the previous case.

Finally, we treat the case in which $\nu(n)=O(1)$ as $n \rightarrow \infty$. In this case, we write

$$
\begin{aligned}
\sum_{\nu=0}^{\infty} a_{n \nu} s_{\nu} & =\sum_{\nu>0} S_{\nu} \Delta^{11} a_{n \nu}=\sum_{\nu>0} \sigma_{\nu}{ }^{*}(\nu+1) \Delta^{11} a_{n \nu} \\
& =\sum_{\nu>(M+1, M+1)}+\sum_{\nu 1}^{M} \sum_{\nu_{2}=M+1}^{\infty}+\sum_{\nu 1}^{\infty} \sum_{\nu_{2}=0}^{M}=\mathrm{I}+\mathrm{II}+\mathrm{III} .
\end{aligned}
$$


It follows from (7.12) and the fact that $\sigma_{\nu} \rightarrow 0$ that $\mid \mathrm{II}<\epsilon$ if $M$ is large enough. Furthermore,

$$
\mathrm{II}=O(1) \sum_{\nu_{1}=0}^{M} \sum_{\nu_{2}=0}^{\infty}\left(\nu_{2}+1\right)\left|\Delta^{11} a_{n \nu}\right|=o(1) \quad \text { by (7.15b). }
$$

Similarly III $=o(1)$.

The proof that (7.11) tends to zero for an arbitrary sequence $\nu(n)$ follows by combining the arguments for the above special cases.

We will complete the proof of Theorem (7.2) by showing that the conditions (7.12)-(7.15) hold. Consider first (7.15a). A calculation shows (see the proof of $[4$, Theorem 1.2, p. 247]) that the summation in (7.15a) is majorized by

$$
\begin{aligned}
& \sum_{\nu_{1}=0}^{\nu_{1}(n)}\left|\Delta^{01} a_{n \nu}\right| g\left(\frac{\nu_{1}}{\nu_{1}(n)+1}, 0\right)+\left(\nu_{1}(n)+1\right) g(0)\left|\Delta^{01} a_{n\left(0, \nu_{2}\right)}\right| \\
& +\sum_{\nu_{1}=0}^{\nu_{1}(n)-1}\left(\nu_{1}(n)-\nu_{1}\right)\left|g\left(\frac{\nu_{1}}{\nu_{1}(n)+1}, 0\right)-g\left(\frac{\nu_{1}+1}{\nu_{1}(n)+1}, 0\right)\right| \cdot\left|\Delta^{01} a_{n \nu}\right| \\
& +\sum_{\nu_{1}=\nu_{1}(n)+1}^{\infty}\left|\Delta^{01} a_{n \nu}\right| g\left(\frac{\nu_{1}(n)+1}{\nu_{1}+1}, 0\right) \\
& +\sum_{\nu_{1}=\nu_{1}(n)+2}^{\infty}\left|\Delta^{01} a_{n \nu}\right|\left(\nu_{1}-1-\nu_{1}(n)\right)\left|g\left(\frac{\nu_{1}(n)+1}{\nu_{1}+1}, 0\right)-g\left(\frac{\nu_{1}(n)+1}{\nu_{1}}, 0\right)\right| .
\end{aligned}
$$

The first and fourth terms combined are less than or equal to

$$
\sup _{k_{1}} \sum_{\nu_{1}=0}^{\infty}\left(a_{n \nu}+a_{n\left(\nu_{1}, \nu_{2}+1\right)}\right) h\left(\nu ; k_{1}, 0\right)
$$

which tends to zero by $(7.5 \mathrm{~b})$. In view of (7.7a), the other terms also tend to zero. Similarly $(7.15 \mathrm{~b})$ holds.

For (7.14) we have ${ }^{*}(\nu(n)+1) a_{n 0} \leqslant \Sigma_{\nu=0}^{\nu(n)+1} a_{n \nu}=O(1)$ by the regularity of $A$.

A calculation like the one done above for (7.15a) will lead to an estimate on the product in (7.13a) whose terms can be bounded by (7.4) and (7.7a). A similar calculation will bound (7.13b) using (7.4) and (7.7b).

We divide the summation in (7.12) into four parts using the point $v(n)$. For the first of these we have 


$$
\begin{aligned}
& \sum_{0<\nu<\nu(n)}{ }^{*}(\nu-\nu(n)) g\left(\frac{\nu+1}{\nu(n)+1}\right) \Delta^{11} a_{n \nu} \\
& =\sum_{\nu=0}^{\nu(n)} a_{n \nu} g\left(\frac{\nu}{\nu(n)+1}\right)+\sum_{\nu_{1}=0}^{\nu_{1}(n)-1} \sum_{\nu_{2}=0}^{\nu_{2}(n)} a_{n \nu}\left(\nu_{1}(n)-\nu_{1}\right) \Delta^{10} g\left(\frac{\nu}{\nu(n)+1}\right) \\
& \quad+\sum_{\nu 1}^{\nu_{1}(n)} \sum_{\nu_{2}=0}^{\nu_{2}(n)-1}\left(\nu_{2}(n)-\nu_{2}\right) a_{n \nu} \Delta^{01} g\left(\frac{\nu}{\nu(n)+1}\right) \\
& \quad+\sum_{\nu=0}^{\nu(n)-1} *(\nu(n)-\nu) a_{n \nu} \Delta^{11} g\left(\frac{\nu}{\nu(n)+1}\right) \\
& \quad+\sum_{\nu_{1}=0}^{\nu_{1}(n)-1}\left(\nu_{1}(n)-\nu_{1}\right)\left(\nu_{2}(n)+1\right) a_{n\left(\nu_{1}, 0\right)}\left(g\left(\frac{\nu_{1}+1}{\nu_{1}(n)+1}, 0\right)-g\left(\frac{\nu_{1}}{\nu_{1}(n)+1}, 0\right)\right) \\
& \quad+\sum_{\nu_{2}=0}^{\nu_{2}(n)-1}\left(\nu_{2}(n)-\nu_{2}\right)\left(\nu_{1}(n)+1\right) a_{n\left(0, \nu_{2}\right)}\left(g\left(0, \frac{\nu_{2}+1}{\nu_{2}(n)+1}\right)-g\left(0, \frac{\nu_{2}}{\nu_{2}(n)+1}\right)\right) \\
& \quad+\left(\nu_{1}(n)+1\right)\left(\nu_{2}(n)+1\right) a_{n 0} g(0) .
\end{aligned}
$$

In this expression, the first term is bounded by (7.4), the second and third are negative, the fourth term is bounded by (7.6), the fifth and sixth terms are bounded because of (7.7) and the seventh can be bounded by (7.4). The other three parts of the summation in (7.12) are treated in a similar manner.

This completes the proof of Theorem 7.2.

8. Localization for the method $L_{1}^{*}$. Let $\Phi(t)=(1+|t|) \log (1+|t|)-|t|$, where $t$ is a real number, and let $\Psi(t)=e^{|t|}-|t|-1$. It is easy to see that these functions are mutually complementary $N$-functions $[1$, p. 14]. We will be interested in the Orlicz spaces $L_{\Psi}^{*}\left(T^{d}\right)$ and $L_{\Phi}^{*}\left(T^{d}\right)$. The space $L_{\Phi}^{*}=L \log ^{+} L$. The space $L_{\Psi}^{*}$ is commonly called the space of exponentially integrable functions and is the space of functions $f$ for which there exists a constant $k>0$ such that $\int_{T^{d}} \exp (k f(x)) d x<\infty$.

We say that a method $[g]$ has the localization property for the space $S$ if $f \in S$ and $f(x)=0$ for $|x|<\rho, \rho>0$, imply that $S_{k}(x, f) \rightarrow 0[g]$ uniformly for $|x|<\rho^{\prime}<\rho$.

THEOREM 8.1. Square order summability $L_{1}^{*}$ has the localization property for the space $L_{\Psi}^{*}$.

Proof. Let $\phi$ be a continuous function on $T^{d}$ such that $\phi(t)=0$ if 
$|t|<\rho$ and $\phi(t)=1$ if $|t|>\delta, t \in T^{d}$, where $\rho$ and $\delta$ are such that $0<\rho<\delta<$ $\pi$. We let $n$ or $m$ denote either integers or $d$-tuples with every entry equal to $n$ or $m$. We will prove that, with $\widetilde{K}_{n, m}(x)=g^{-1}(m /(n+1)) \bar{K}_{n, m}(x)$,

$$
\sup \left\{\left|\sigma_{n, m}^{1}(x, \phi f)\right|:|x|<\rho^{\prime}, 0 \leqslant m \leqslant n\right\} \leqslant A\|f\|_{\Psi}
$$

where $0<\rho^{\prime}<\rho$. The usual argument using a dense class in $L_{\Psi}$ and (8.1) yields the theorem. To begin, note that an application of Hölder's inequality for Orlicz spaces yields

$$
\left|\sigma_{n, m}^{1}(x, \phi f)\right| \leqslant\left\|\phi(x+t) \widetilde{K}_{n, m}(t)\right\|_{\Phi} \cdot\|f\|_{\Psi} / \pi^{d} .
$$

By $[1$, p. 222(20)]

$$
\left\|\phi(x+t) \widetilde{K}_{n, m}(t)\right\|_{\Phi} \leqslant 1+\int_{T^{d}} \Phi\left(\phi(x+t) \widetilde{K}_{n, m}(t)\right) d t .
$$

Let $\eta=\left(\rho-\rho^{\prime}\right) / \sqrt{ } d$. Note that $|x|^{\prime}<\rho$ and $|t|<\rho-\rho^{\prime}$ implies $|x+t|<\rho$ and that $|t|<\rho-\rho^{\prime}$ if $\left|t_{i}\right|<\eta$ for all $i=1,2, \cdots, d$. Thus $\phi(x+t)=0$ if $\left|t_{i}\right|<\eta$ and $|x|<\rho$. It follows that

$$
\begin{aligned}
\int_{T^{d}} \Phi\left(\phi(x+t) \widetilde{K}_{n, m}(t)\right) d t & \leqslant C \int_{0}^{\pi} \cdots \int_{0}^{\pi} \int_{\eta}^{\pi} \Phi\left(\widetilde{K}_{n, m}(t)\right) d t \\
& \leqslant C\left(1+\int_{0}^{\pi} \cdots \int_{0}^{\pi} \int_{\eta}^{\pi}\left|\widetilde{K}_{n, m}(t)\right| \log ^{+}\left|\widetilde{K}_{n, m}(t)\right| d t\right) .
\end{aligned}
$$

The last inequality follows from the fact that $\Phi(t) \leqslant t \log ^{+} t$ if $t \geqslant 10$. Since $\log ^{+}|u v| \leqslant \log ^{+}|u|+\log ^{+}|v|$ we obtain

$$
\begin{aligned}
\int_{0}^{\pi} \int_{0}^{\pi} \cdots & \int_{\eta}^{\pi}\left|\widetilde{K}_{n, m}(t)\right| \log ^{+}\left|\widetilde{K}_{n, m}(t)\right| d t \\
& \leqslant \sum_{j=1}^{d} \int_{0}^{\pi} \cdots \int_{0}^{\pi} \int_{\eta}^{\pi}\left|\widetilde{K}_{n, m}(t)\right| \log ^{+}\left|\widetilde{K}_{n, m}\left(t_{j}\right)\right| d t
\end{aligned}
$$

where

$$
\widetilde{K}_{n, m}\left(t_{j}\right)=\left(1+\log \left(\frac{n+1}{n-m+1}\right)\right)^{-1} K_{n, m}\left(t_{j}\right)
$$

By using the estimates (2.3), (2.4) and (2.5) and the notation $\alpha=(n+1+m)^{-1}$ and $\beta=(n+1-m)^{-1}$ and splitting the integral $\int_{0}^{\pi}$ into three parts $\int_{0}^{\pi}=$ $\int_{0}^{\alpha}+\int_{\alpha}^{\beta}+\int_{\beta}^{\pi}$, it is easily seen that

$$
\int_{0}^{\pi}\left|\widetilde{K}_{n, m}\left(t_{i}\right)\right| d t_{i} \leqslant C
$$

for all $i$. Furthermore, by (2.5) 


$$
\int_{\eta}^{\pi}\left|\widetilde{K}_{n, m}\left(t_{i}\right)\right| d t_{i} \leqslant C \beta(1+\log \beta(n+1))^{-1} \leqslant C(\log (n+1))^{-1}
$$

and

$$
\int_{\eta}^{\pi}\left|\widetilde{K}_{n, m}\left(t_{j}\right)\right| \log +\widetilde{K}_{n, m}\left(t_{j}\right) \mid d t_{j} \leqslant C
$$

This shows that (8.2) is majorized by

$$
C(\log (n+1))^{-1} \int_{0}^{\pi}\left|\widetilde{K}_{n, m}(t)\right| \log ^{+}\left|\widetilde{K}_{n, m}(t)\right| d t+C
$$

Again, using (2.3), (2.4) and (2.5), we obtain

$$
\begin{aligned}
& \int_{0}^{\pi}\left|\widetilde{K}_{n, m}(t)\right| \log { }^{+}\left|\widetilde{K}_{n, m}(t)\right| d t \\
& \leqslant C\left(1+\log \left(\frac{n+1}{n+1-m}\right)\right)^{-1}\left[\int_{0}^{\alpha} \alpha^{-1} \log ^{+} A \alpha^{-1} d t+\int_{\alpha}^{\beta} \log ^{+} A t^{-1} \frac{d t}{t}\right. \\
&\left.\quad+\int_{\beta}^{\pi} \beta^{-1} \log ^{+}\left(\beta^{-1} A t^{-2}\right) \frac{d t}{t^{2}}\right] \\
& \leqslant C\left(1+\log \frac{n+1}{n+1-m}\right)^{-1}\left[1+\log (n+1)+\log \frac{n+1}{n+1-m}+\log (n+1)\right] .
\end{aligned}
$$

This estimate combined with (8.3) gives (8.1).

\section{REFERENCES}

1. M. A. Krasnosel'skĩ and Ja. B. Rutickiir, Convex functions and Orlicz spaces, Problems of Contemporary Mathematics, GITTL, 1958; Fizmatgiz, 1959; English transl., Noordhoff, Groningen, 1961. MR 21 \#5144; 23 \#A4016.

2. S. Igari, On the localization property of multiple Fourier series, J. Approximation Theory 1 (1968), 182-188. MR 40 \#641.

3. W. B. Jurkat and A. Peyerimhoff, Fourier effectiveness and order summability, J. Approximation Theory 4 (1971), 231-244. MR 43 \#7855.

4. - Inclusion theorems and order summability, J. Approximation Theory 4 (1971), 245-262. MR 43 \#7856.

5. J. Marcinkiewicz and A. Zygmund, On the summability of double Fourier serles, Fund. Math. 32 (1939), 122-132.

6. J. Marcinkiewicz, B. Jassen and A. Zygmund, Note on the differentiability of multiple integrals, Fund. Math. 25 (1935), 217-234.

7. E. M. Stein and N. J. Weiss, On the convergence of Poisson integrals, Trans. Amer. Math. Soc. 140 (1969), 35-54. MR 39 \#3024.

8. A. Zygmund, Trigonometric series, 2nd ed., Cambridge Univ. Press, New York, 1959. MR 21 \#6498.

DEPARTMENT OF MATHEMATICAL SCIENCES, UNIVERSITY OF MISSOURI, ST. LOUIS, MISSOURI 63121 